\title{
Suitability of Infrared Thermography for Monitoring the Hot Extrusion of Insulating Materials
}

\author{
by J. Aderhold*, P. Meinlschmidt*, F. Schlüter*
}

\begin{abstract}
*Fraunhofer Institute for Wood Research, Department VST, Bienroder Weg 54 E, 38108 Braunschweig,
\end{abstract} Germany, jochen.aderhold@wki.fraunhofer.de

\section{Abstract}

This work presents first results of research aiming at the inline measurement of thermal conductivity of insulation materials produced by hot extrusion. Samples of insulation material were heated by laser pulses or cooled by cooling fingers. The resulting hot or cold spots were tracked, and their infrared intensities were recorded. These are a measure of the complex time dependency of the temperature in the excited areas. After an initial phase presumably characterized by the sample's semitransparency, the intensities follow a well-understood proportionality to $\mathrm{t}^{-0.5}$ at least for some time, which allows the extraction of the thermal conductivity by fitting.

\section{Introduction}

Hot extrusion is a widespread production process for plastics products with a cross-section that is constant lengthwise. The raw material is melted and pushed through the extrusion tool with a speed in the range of one metre per minute. Extrusion gives a very smooth surface and is able to process brittle materials such as expanded polystyrene (XPS). However, changes in the composition of the raw materials (for example caused by use of recovered plastics) and variations in temperature and pressure can result in structural defects such as air inclusions or in an inhomogeneous distribution of material parameters such as thermal conductivity.

When the product leaves the extrusion tool, heat is dissipated by heat flow from the interior to the surfaces. The heat flow pattern in defective areas is different from that in good parts of the structure, if the defects differ in thermal conductivity and/or thermal capacity from the faultless material. This is especially true for air inclusions and density fluctuations. The different heat flows are mirrored in the temperature distribution on the product's surface. These temperature patterns and consequently the defects can be made visible with an infrared camera (thermography). Since infrared thermography is an imaging technique, many established procedures and algorithms from conventional image processing for automatic defect recognition can be adopted.

Furthermore, the thermal conductivity of the material can be characterized by means of thermography if it is subjected to a sequence of laser pulses. The laser pulses heat up the insulating material locally and generate a characteristic temperature profile. After the pulse, horizontal and vertical heat flows broaden the temperature profile and decrease its peak height.

In a joint project called "Sensoren und Auswertestrategien zur autonomen Überwachung von kontinuierlichen Kunststoffprozessen" (KontiSens), the Fraunhofer Institute for Chemical Technology (ICT), the WKI, and partners from industry work on the development of sensors and evaluation strategies for the autonomous monitoring of continuous plastics production processes. The task of the WKI is to develop a monitoring technology based on infrared imaging for the production of insulating materials by hot extrusion.

\section{State of the Art}

The measurement of thermal conductivities of insulating materials is regulated in various national and international standards $[1,2,3]$. In all standard procedures, one or more plate-shaped samples are positioned between at set-up of heating and cooling plates. The thermal conductivity is calculated from the temperature difference between heating and cooling plates and from the heat flow. The latter is either estimated from the electric power fed into the heating plate(s) or from calibration measurements using reference samples with known thermal conductivity. Due to the time necessary for the establishment of thermal equilibrium, these measurements last some hours or some days.

Transient methods [4] work much faster, but are not suitable for moving samples and are thus not a choice for direct process control.

Active heat-flow thermography is a non-destructive inspection method basing on a visualization of differences in thermal properties. It is easy to integrate into industrial manufacturing processes. It is an obvious idea to modify the method in such a way that the thermal conductivity can be measured if the thermal capacity is known. In this work, an infrared camera was used to measure surface temperatures. The necessary thermal excitation was done either with a positive sign (by laser irradiation) or with a negative sign (by cooling fingers). While there is much information on inline heat flow thermography as a technique for finding differences in thermal properties ([5,6] and references therein), the quantitative measurement of thermal properties by this method is mostly limited to offline applications (see [7] as an example). 


\section{Theory}

After a short introduction to heat flow thermography (4.1), the following paragraphs describe some ways to obtain the thermal conductivity of a material from the measured infrared intensities of the thermally excited spots as a function of time.

From laser material machining the relationship between laser power and the maximum of the surface temperature is known, taking into account the laser beam profile, but not considering its time dependency (4.2). Surface temperature as a function of time was studied extensively, especially for two limiting cases: the homogenous excitation of a semi-infinite plane and point-like excitation, respectively. The first case is a good approximation for a situation where the horizontal dimensions of the plane are large compared to the thermal diffusion length of the material. In the opposite situation, pointlike excitation is a good assumption. Since the thermal diffusion length is proportional to the square root of time, both limiting cases can be considered as approximations for laser heating of a plane surface which develop subsequently in the course of time (4.3). These models do not consider the real spatial profile of the laser beam.

\subsection{Heat flow thermography}

Thermal conduction in solids without internal heat sources is described by Fourier's law

$$
\nabla(\nabla \kappa T))=\frac{\partial T}{\partial t}
$$

where $t$ is the time, $T$ the (absolute) temperature and $\kappa$ the thermal diffusivity defined by

$$
\kappa=\frac{\lambda}{\rho C_{S p}}
$$

using the thermal conductivity $\lambda$, the mass density $\rho$ and the specific heat capacity $C_{s p}$

A transient heat flow within an object will cause a transient temperature pattern at the surface, which represents the distribution of thermal diffusivity. If one images the temperature pattern with an infrared camera, differences in thermal conductivity and/or thermal capacity can be detected with a certain penetration depth, which depends on the material. In such a way, structural defects in an object can be found.

The heat flow can be induced by active thermal excitation (active heat flow thermography) or simply by utilizing the cooling-down of an object which was heated in a preceding production step (passive heat flow thermography). The inspection of material coming out of an extruder is an example for the passive method.

\subsection{Temperature rise caused by laser scanning}

When an object is exposed to a laser beam with diameter $d$ and beam profile $I(x, y)$ moving in $x$ direction with speed $\mathrm{v}_{0}$, the maximum temperature rise at the surface $\Delta T_{\max }$ is given by [8]

$$
\Delta T_{\max }=\frac{\varepsilon}{2 \lambda \pi d} \iint_{A} \frac{I(u, v) e^{-P_{n}[r-(x-u)]}}{r} d u d v
$$

where $\varepsilon$ is the emissivity of the material (=absorptivity for opaque materials). The Péclet number $P_{n}$ is a dimensionless quantity specifying the relation of convective and conductive heat transport, respectively. It is defined by

$$
P_{n}=\frac{2 d v_{0}}{\kappa}
$$

The dimensionless quantities $u$ and $v$ are the spatial co-ordinates $x$ and $y$ divided by the beam diameter $d$. The denominator of the integrand is

$$
r=\sqrt{(x-u)^{2}-(y-v)^{2}} .
$$

One can easily see that $\Delta T_{\max }$ is essentially inversely proportional to the thermal conductivity. If all other parameters are kept constant, a monitoring of the maximum temperature is sufficient to detect fluctuations in thermal conductivity.

\subsection{Evaluation of the time dependency of temperature}

Another way to monitor the thermal conductivity of the extruded material is to observe the development of temperature over time. For a semi-infinite plane and a spatially homogeneous, temporally Dirac-like heat pulse with an energy per area $Q_{0}$, the temperature rise at distance $x$ from the surface is given by [9]: 


$$
\Delta T(t)=T(t)-T(t=0)=\frac{Q_{0}}{2 \sqrt{\pi \rho C_{S p^{\kappa t}}}} \exp \left(\frac{-x^{2}}{4 \kappa t}\right)
$$

For $\mathrm{t}$ approaching zero this function approaches the Dirac function except for a multiplicative factor (delta distribution). For the surface $(x=0)$ und $t>0$ the temperature difference is essentially proportional to the square root of time. In a logarithmic plot, a straight line with slope -0.5 is the result. The intercept contains $Q_{0}, \rho, C_{s p}$ and $\kappa$. If all other quantities are known, $\kappa$ and thus the thermal conductivity can be calculated.

In case the heat pulse is spatially point-like, a similar relation is valid, but with slope -1.5 [10]. Whether a laser beam is to be regarded as spatially homogenous or spatially point-like, depends on the relation of its diameter $d$ to the thermal diffusion length $\mu$ given by

$$
\mu=2 \sqrt{\kappa t} .
$$

In the case $d>>\mu$, the laser can be regarded as spatially homogenous. For the case $d<<\mu$ it is rather a point-like excitation. Since the thermal diffusion length increases with time, the temperature as a function of time will at first have a slope of -0.5 in a logarithmic plot (homogenous excitation). In the course of time, with increasing $\mu$, the absolute value of the slope will increase and finally reach an asymptotic value of -1.5 .

In the case of a semi-transparent material with an optical absorption coefficient $\beta$, the temperature difference as a function of time for spatially homogenous excitation is given by

$$
\Delta T(t) \sim \exp \left(\beta^{2} \kappa t\right) \cdot \operatorname{erfc}(\beta \sqrt{\kappa t})
$$

where erfc is the complementary error function [11]. This can be approximated for large arguments as

$$
\operatorname{erfc}(x)=x^{-1} \pi^{-\frac{1}{2}} \exp \left(-x^{-2}\right) \cdot\left[1-\frac{1}{2} x^{-2} \ldots\right]
$$

For slightly transparent materials (with a large absorption coefficient) one obtains

$$
\Delta T(t) \sim \frac{1}{\beta \sqrt{\pi \kappa t}}\left[1-\frac{1}{2 \beta \kappa t} \ldots\right]
$$

Asymptotically again a proportionality to the square root of time results, while the temperature difference at the beginning is smaller than for opaque materials.

\section{Experiments}

First tests took place in co-operation with Laser Zentrum Hannover e. V. using commercially available XPS samples at room temperature. The aim of the test was to find out if the temperature profiles caused by laser beams can be imaged by an infrared camera and automatically extracted by image processing with sufficient precision, and if the development of the temperature profiles over time meets the theory.

A pulsed solid-state laser with a wavelength of $960 \mathrm{~m}$ and a maximum power of $25 \mathrm{~W}$ served as the excitation source. In order to simulate the transport of the XPS out of the extruder, a conveyor belt moved them at a speed of 3 metres per minute perpendicularly to the laser beam. Pulse rate and spot diameter amounted to $1 \mathrm{~Hz}$ and $1 \mathrm{~cm}$, respectively. An infrared camera from IRCAM GmbH (Erlangen, Germany) recorded up to five laser-heated spots at the same time at a frame rate of $25 \mathrm{~Hz}$. The camera's InSb detector had 512 × 640 pixels and a NETD of 15 mK. See figure 1 for details of the set-up.

The amount of absorbed heat depended strongly on the colour of the samples, which in turn is manufacturer specific. White samples showed a maximum temperature rise of only $2.5 \mathrm{~K}$, while greenish samples already melted in the spot centre using the same laser parameters.

Thermal excitation by laser is simple and contact-free. However, the strong dependency of laser absorption on sample colour is a drawback. Furthermore, heating an already hot material could be a problem and lead to damage of the samples. For these reasons, additional tests with thermal excitation with a negative sign, that means a point-like coolingdown of the sample, were carried out. Rounded steel rods with a diameter of $8 \mathrm{~mm}$, cooled down to $-20^{\circ} \mathrm{C}$ with a RanqueHilsch vortex tube and pressed manually against the samples, served as the excitation sources. These tests used the pilot extruder of Fraunhofer ICT in Pfinztal (figure 2). Besides the test of the excitation method, the aim of these experiments was to see if structural inhomogeneities in the extruded material are detectable by passive heat flow thermography.

\section{Image Processing for Data Extraction}

In order to evaluate the temperature changes induced by laser irradiation or cooling fingers, the excited areas had to be located in the images, and their intensities had to be extracted. In an industrial application, this has of course to 
be automated. Consequently, an image-processing algorithm for this task had to be developed. It is described here for laser excitation. For the cooling method, the same algorithm can be used after inverting the images.

For reasons of robustness, the algorithm had to obtain as many information as possible from the image series themselves. Only the following assumptions were made:

- $\quad$ The excited areas are more intense than their immediate vicinity, but not necessarily the most intense regions in the images.

- $\quad$ The excited areas move in one direction with constant speed and distance.

- The excited areas are more or less round. Technically speaking, their eccentricity is much smaller than unity.

A typical infrared image with four laser-heated spots is shown in figure 3 . The laser spots are clearly visible, and their intensities decrease in transport direction (here: from left to right), as expected.

Series of 250 images each were evaluated, representing a time span of ten seconds. In a first step, the mean over all images was calculated und subtracted from the series in order to remove the background. A typical result is shown in figure 4 . In the image region where the spots move, the intensities oscillate as a function of time, whereas they are constant in the rest. This fact can be used for identifying that part of the images where the spots can be found.

Principal Component Analysis (PCA) served as a tool for finding the differences in the time dependency of the intensities. The third principal component was found to be most suitable for detecting a region of interest that contains the spots. In figure 5, this is a strip containing the black and white dots. The remaining part of the image (light grey in figure 5) is not of interest anymore and can be cut off.

The next step is to segment the spots by a simple intensity threshold calculated by the Otsu method [12]. Possible remaining objects with high intensity that are not spots can be eliminated by deleting all objects with an eccentricity larger than the average eccentricity. (The eccentricity is a measure for the "roundness" of an object. A circle has an eccentricity of 0 , and a straight line has an eccentricity of 1.) Afterwards, a morphological opening is applied, and objects with a size falling below the average size are removed.

In such a way, the algorithm finds almost every spot. A linear fit between the calculated positions of the spots and the frame number (representing the time) helps to identify possible outliers since the spots are expected to move at constant speed. Finally, the intensities of the spots are extracted in two dimensions and saved for further evaluation.

\section{Results}

At first, an example for qualitative heat flow thermography for the inspection of structural quality of the extruded material will be shown. This is a result from simply imaging the material passively without any external excitation and sort of a by-product of the quantitative measurements.

The evaluation of the maximum temperature rise at the surface as a measure for the thermal conductivity as described in chapter 4.2 was not made for the time being because the laser beam profile proved to be unstable. Hence, equation 2 could not be applied. Thus, only the dependency of intensities over time as described in chapter 2 was considered.

A typical result from passive thermography behind the extruder can be seen in figure 6 . The apparent inhomogeneities vanished with the establishment of thermal equilibrium. Thus, different intensities are a sign of different surface temperatures and hence of different thermal properties caused by structural inhomogeneities and not a sign of different emissivities.

Comparing theory with measurement, one has to keep in mind that the infrared camera records intensities, while the theory makes predictions of temperatures. The relation between these two quantities is showcased in figure 7 . It is not linear but can be linearized with little loss of precision. Thus, in the following intensity and temperature will be used as synonyms.

Figure 8 shows a typical intensity profile for a laser heated spot. The left part shows raw data, the right part a smoothed profile. Typical spot intensities as a function of time for "white" material can be seen in figure 9. The curves for four different spots do not coincide. This is most likely a consequence of fluctuating laser intensity. About ten seconds after the excitation, the curves meet the theoretical expectation. In a logarithmic plot, they follow straight lines with slopes in range of -0.5 .

As figure 10 shows, the absolute value of the slope increases continuously after the pulse and then assumes the theoretical value for the excitation of a semi-infinite plane. This means that the thermal diffusion length is still smaller than the diameter of the laser beam. The initially smaller absolute value of the slope is likely a consequence of the semitransparency of the material in the wavelength range where the camera is sensitive (see equation 10).

Figure 11 shows intensities as a function of time for "green" material. The absolute values of the asymptotic slopes are larger than for "white" material. As mentioned before, the amplitude of temperature is much larger here. This led to a partial melting in the centre of the laser spot. Consequently, the theory drafted in chapter 2 possibly does not apply.

The dependency of intensities on time for the excitation with cooling fingers using "white" material shows a similar behaviour as those caused by laser heating, only with reversed sign (figure 12). Consequently, their asymptotic slopes are in the range of +0.5 . The differences between the single curves are even larger than for laser heating which is not surprising since the cooling fingers were pressed against the samples manually. 


\section{Conclusion and Outlook}

It was shown that structural inhomogeneities in extruded XPS can be detected by passive heat flow thermography. Furthermore, the feasibility of automated extraction of intensity profiles as a function of time caused by either laser heating or by pressing cooling fingers against the material was demonstrated. For moderate laser intensities and for the cooling fingers they meet the theoretical expectations.

In the subsequent phases of the project, a machine for an automated and reproducible application of the cooling fingers (figure 13) will be tested, and thermal properties of the samples will be measured by standard methods and compared with the results from thermography.

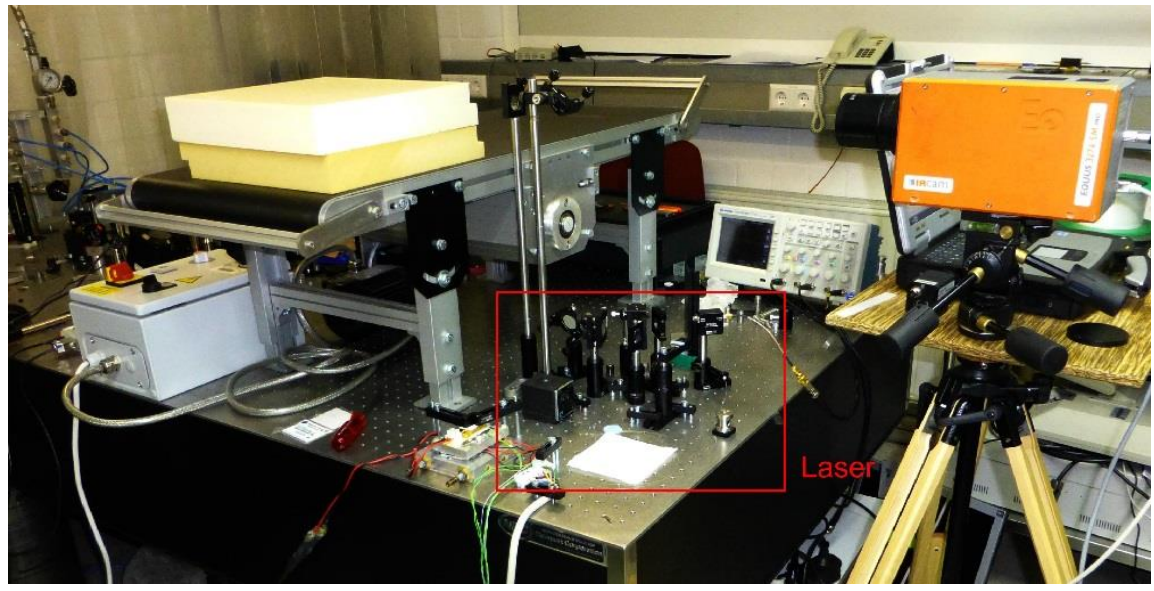

Fig. 1: Set-up for the first experiments

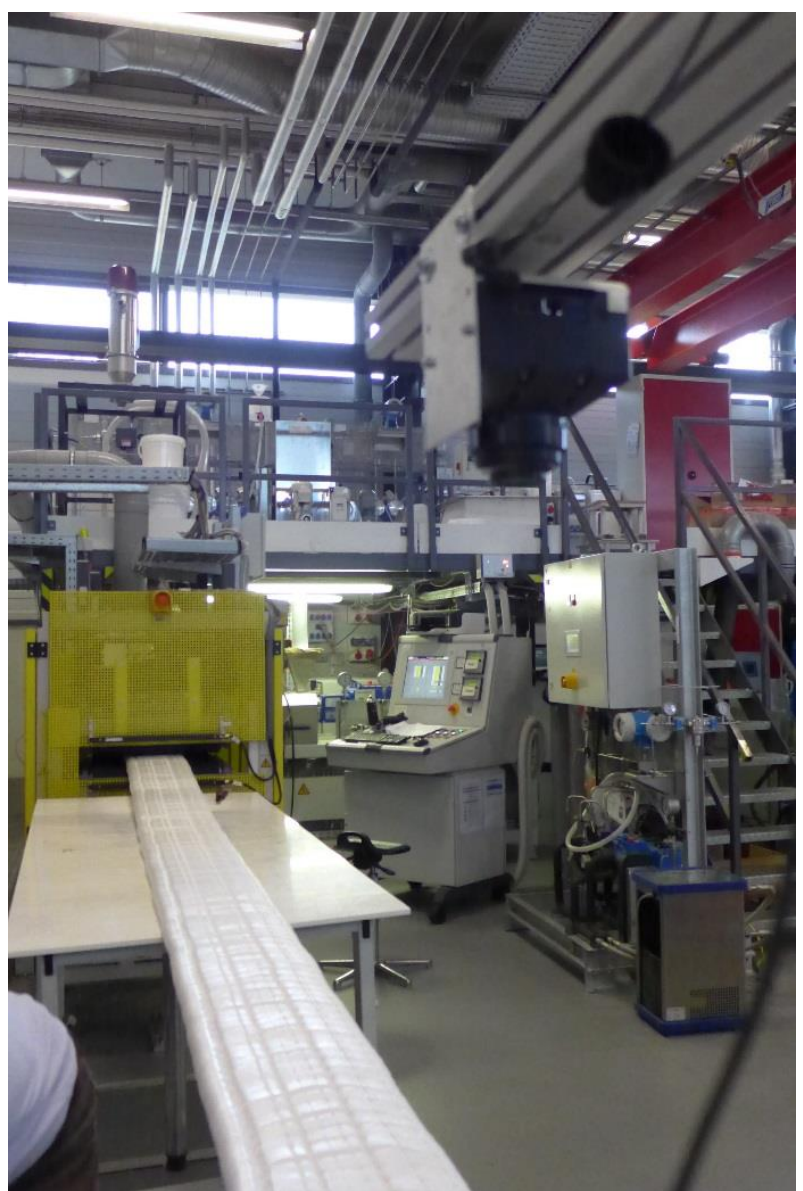

Fig. 2: Passive thermography at the pilot extruder of Fraunhofer ICT 


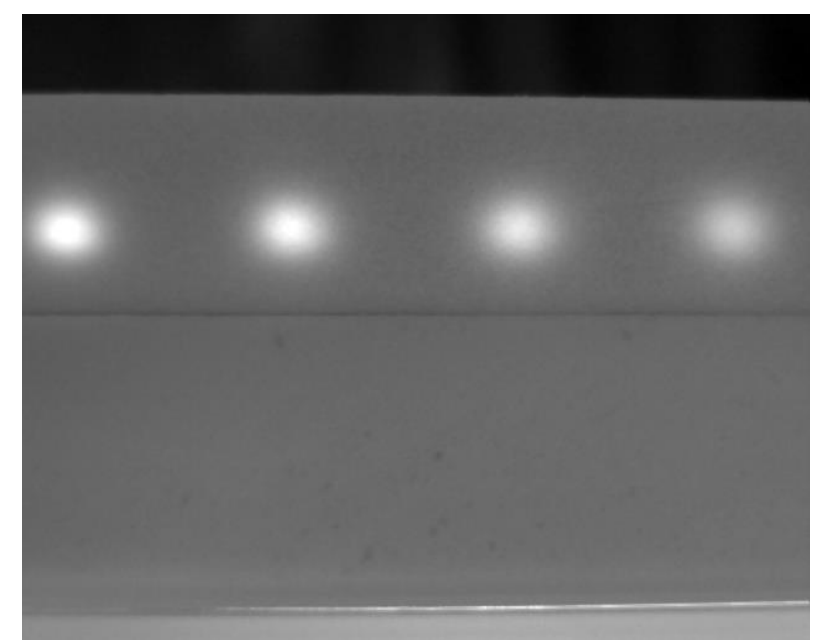

Fig. 3: Typical infrared image with four laser spots

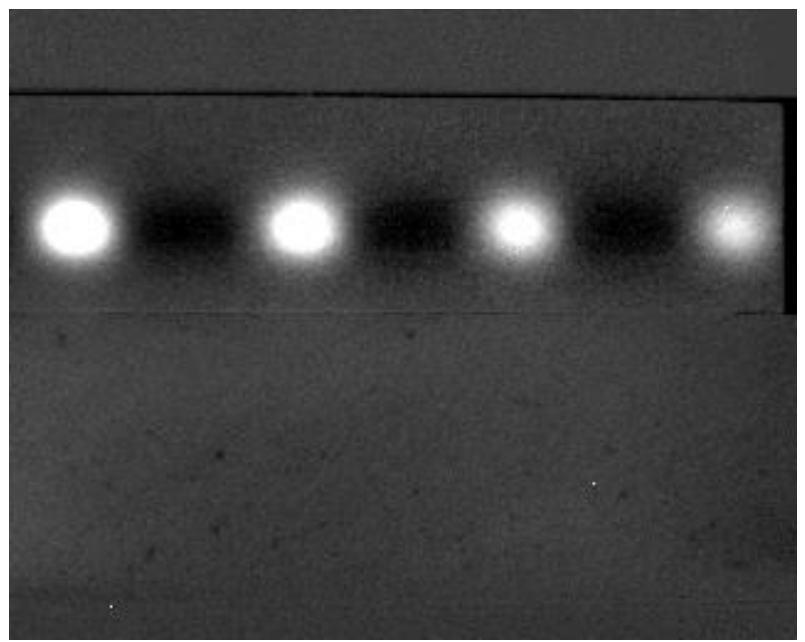

Fig. 4: Typical infrared image after background removal

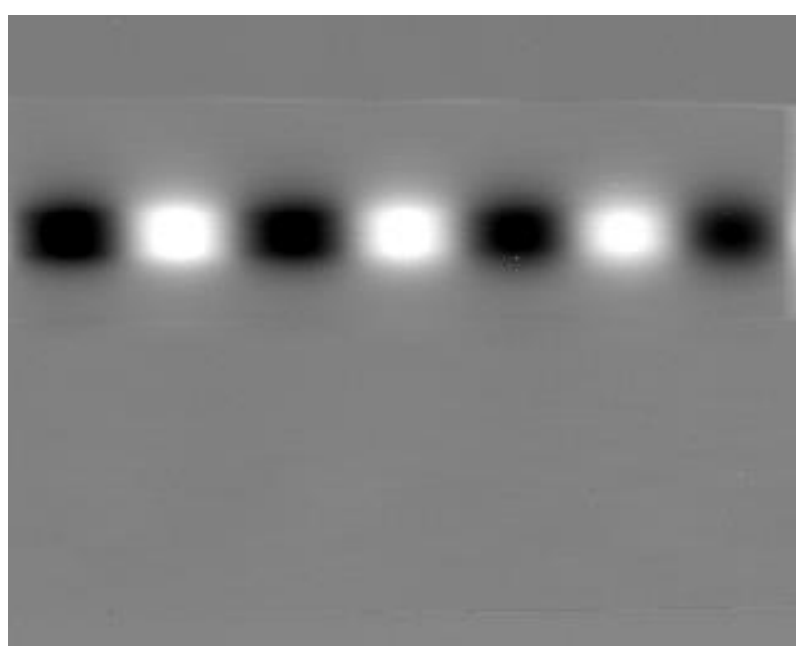

Fig. 5: Image representation of the third principal component of I(t) for every pixel 


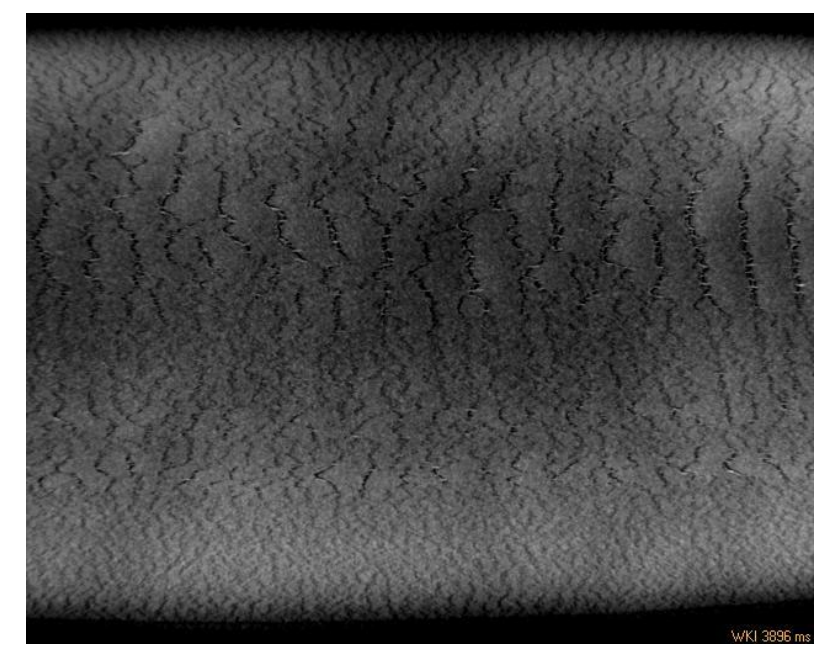

Fig. 6: Infrared image of structural inhomogeneities of the extruded material

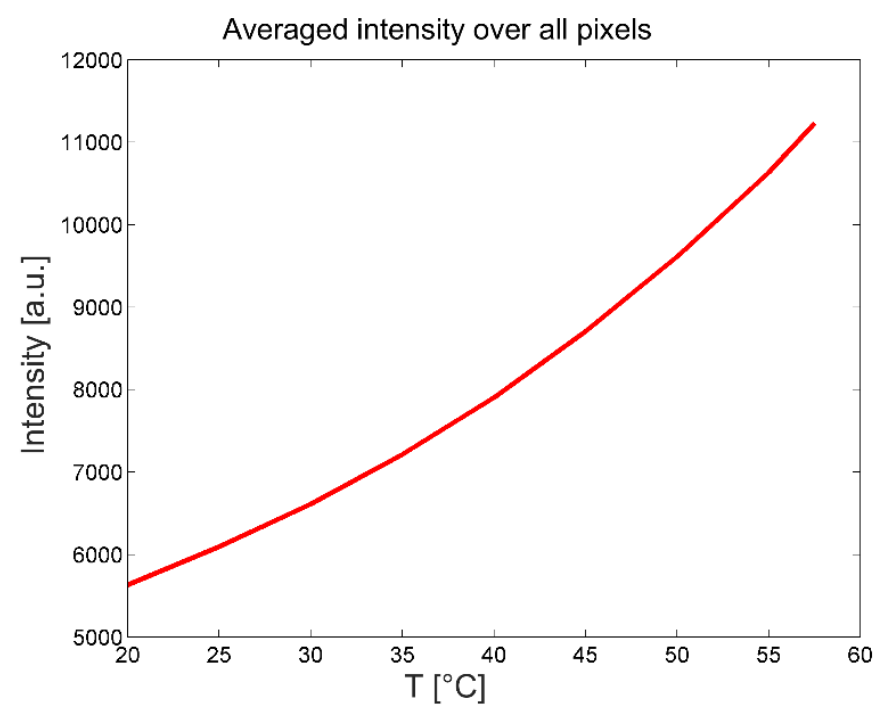

Fig. 7: Typical dependency of intensity on temperature for an infrared camera

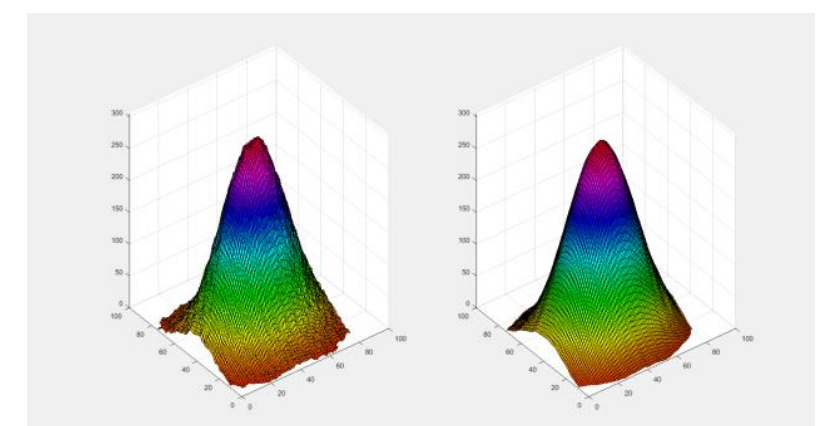

Fig. 8: Typical shape of a laser spot. Left: raw data, right: smoothed data 


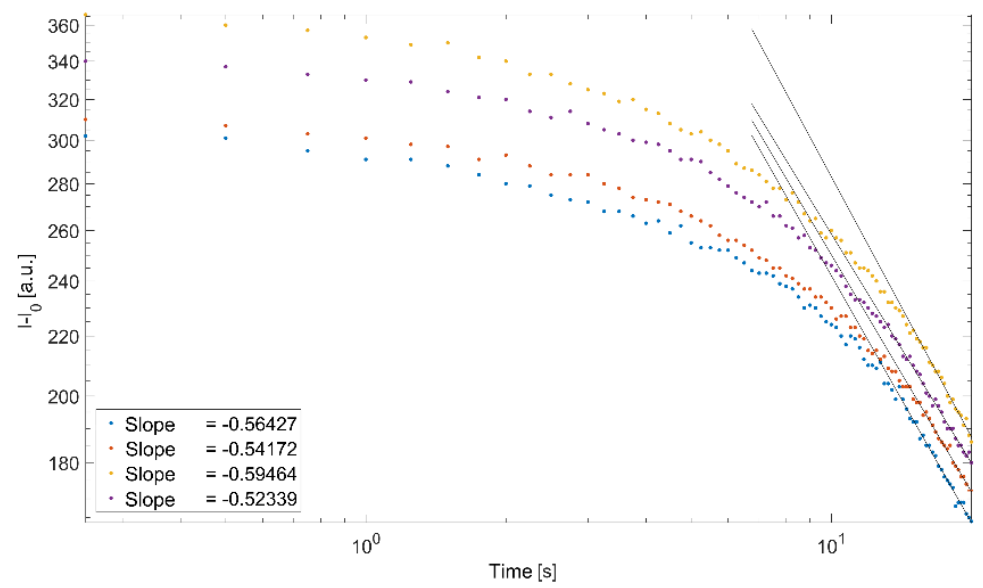

Fig. 9: Typical intensities at the laser heated spots as a function of time for "yellow" material
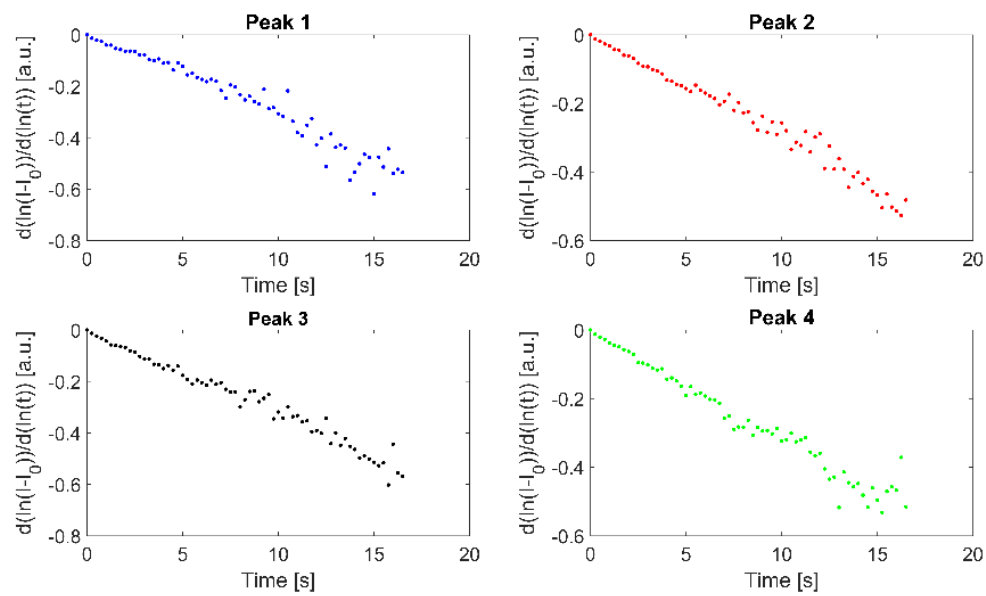

Fig. 10: Slope of logarithmic plot of I(t)-lo for different laser spots for "yellow" material

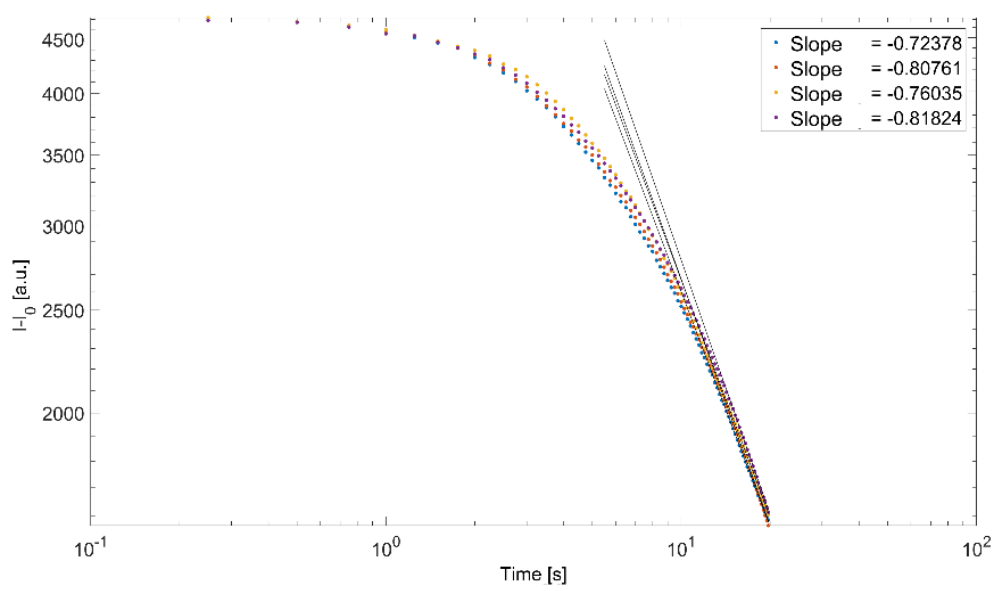

Fig. 11: Typical intensities at the laser heated spots as a function of time for "green" material 


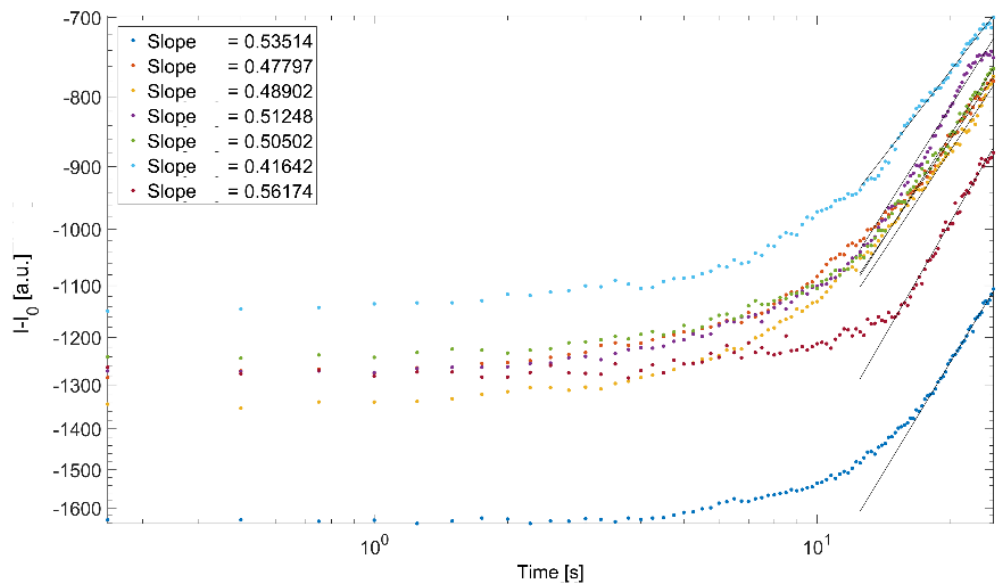

Fig. 12: Typical intensities at the spots cooled by cooling fingers as a function of time
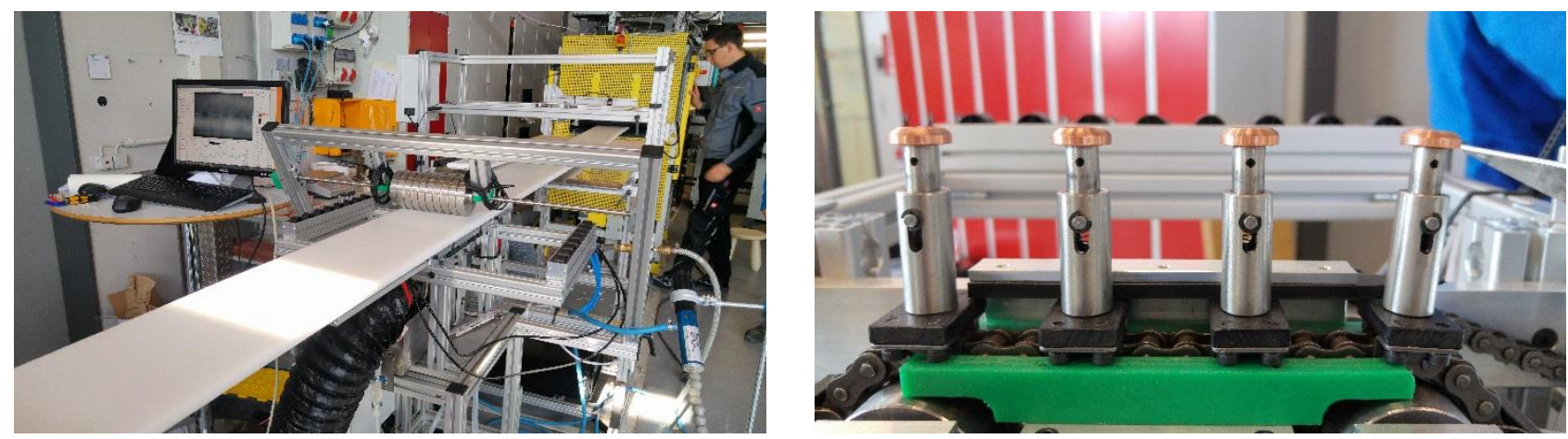

Fig. 13: Machine for an automated and reproducible application of the cooling fingers

\section{REFERENCES}

[1] DIN e. V. (2001). Bestimmung des Wärmedurchlasswiderstandes nach dem Verfahren mit dem Plattengerät und dem Wämestrommessplatten-Gerät. Dicke Produkte mit hohem und mittlerem Wärmedurchlasswiderstand. Berlin, Beuth-Verlag. DIN EN 12939

[2] DIN e. V. (2001). Bestimmung des Wärmedurchlasswiderstandes nach dem Verfahren mit dem Plattengerät und dem Wärmestrommessplatten-Gerät. Produkte mit hohem und mittlerem Wärmedurchlasswiderstand. Berlin, Beuth-Verlag. EN 12667

[3] ISO (1991). Thermal insulation - Determination of steady-state thermal resistance and related properties. Heat flow meter apparatus. Geneve, ISO. ISO 8301

[4] Hammerschmidt, U. and V. Meier (2006). "New Transient Hot-Bridge Sensor to Measure Thermal Conductivity, Thermal Diffusivity, and Volumetric Specific Heat." International Journal of Thermophysics 27(3): 840-865

[5] Vavilov, V. P. and D. D. Burleigh (2015). "Review of pulsed thermal NDT: Physical principles, theory and data processing." NDT \& E International 73: 28-52

[6] Balageas, D., X. Maldague, et al. (2016). "Thermal (IR) and Other NDT Techniques for Improved Material Inspection." Journal of Nondestructive Evaluation 35(1): 1-17

[7] Greppmair, A., B. Stoib, et al. (2017). "Measurement of the in-plane thermal conductivity by steadystate infrared thermography." Review of Scientific Instruments 88(4): 044903

[8] G. R. B. E. Römer and J. Meijer, Metal surface temperature induced by moving laser beams, Optical and quantum electronics, vol. 27, no. 12, pp. 1397-1406, 1994

[9] D. P. Almond and P.-M. Patel, Photothermal Science and Techniques. London, UK: Chapman and Hall, 1996 
[10] H. S. Carslaw, J. C. Jaeger, Conduction of Heat in Solids. Oxford: Clarendon Press, 1993

[11] Imhof, R., D. Birch, et al. (1984). "Optothermal transient emission radiometry." Journal of Physics E: Scientific Instruments 17(6): 521

[12] Otsu, N. (1979). "A Threshold Selection Method from Gray-Level Histograms." IEEE Transactions on Systems, Man and Cybernetics SMC-9(1): 62-66 